\title{
Role of Artificial Intelligence in Social Media and Human Behaviour
}

\author{
Smitarani Satpathy, Srikanta Patnaik
}

\begin{abstract}
The paper contains the simple idea of Artificial Intelligence on social media and human behaviour. The major concern of this paper is to show the changing behaviour of human being and Artificial Intelligence usefulness in human life as well as social media. Social media popularity came into high during the last decade due to smart technologies used in mobile and internet. In last 10 years research on AI shows its impact on human life as well as in media marketing.
\end{abstract}

Keywords: Artificial Intelligence (AI), Social Media, Analytics, Facebook (FB).

\section{INTRODUCTION}

As modern technology comes, different tools and technological instrument implemented to attract the customers over social media. AI is making a best journey for the user by leaking the experience on social network of the user. The best example of AI is Facebook, uses neural network to image recognition for its users; they can find their friends easily on social sites. Most of the users would be surprised how it works? The basic aim of the study is to make more efficient service to the user. The companies are also using AI for filtering the data of the user and for the benefit of social media in business. Using it in business, could be better techniques to understand the buying behaviour of the customer and implement some more effective strategies for their business to motivate the user. The method deep learning is going to help people in searching from the keyword. The faster changing social media environment makes hurdles for the top brand companies. The converted information of those social media data can analyze through Machine learning algorithms and marketers can utilize that information in their business. AI is empowered by Deep learning, algorithms and datasets. The meaning of artificial intelligence is investigating intelligent problem-solving behaviour and creating intelligent computer systems. The Machine Learning and Natural Language Processing, methodologies present to find the misinformation to some extent.

Manuscript received on October 13, 2021.

Revised Manuscript received on October 24, 2021.

Manuscript published on October 30, 2021.

*Correspondence Author

Smitarani Satpathy*, Department of Computer Science and Engineering, SOA (deemed to be) University, Bhubaneswar (Odisha), India. E-mail. smitasatpathy.bbsr@gmail.com

Dr. Srikanta Patnaik, Department of Computer Science and Engineering, SOA (deemed to be) University, Bhubaneswar (Odisha), India. E-mail. srikantapatnaik@soa.ac.in

(C) The Authors. Published by Blue Eyes Intelligence Engineering and Sciences Publication (BEIESP). This is an open access article under the CC BY-NC-ND license (http://creativecommons.org/licenses/by-nc-nd/4.0/)
But, existence of semantic nature of data, the precision of automated methods is confined [1]. AI is to be known to all that $\mathrm{AI}$ is the most useful topic in human life. There are so many example of AI like cortana by windows mobile, siri by apple, Watson by IBM etc. for different o/s which are intelligent digital personal assistants [2][3] which helps in problem solving solution and searching things for human without physical appearance. They can only work through commands. Recent research on humanoid means machines with emotions and feelings like human Roborts by the scientists. The development of cars without driver is an example of this type of Robort. Waymo[4] is a self-driving car.

\section{TYPES OF ARTIFICIAL INTELLIGENCE IMPLEMENTATION}

1. Using Chatbots \& Virtual Assistance: Companies are benefited through chatbots due to its assumption reduction process and helped the marketers for the analysis on the basis of customer needs and interest.

2. Optimizing content for social sites: It helps in brand awareness and sales of product through the Virtual Assistance or Chatbots. The intelligent based system helps the marketers to optimize their content for sales and marketing.

3. Predicting the Outgrowth: The queries related to product may manage through Machine Learning algorithm. For example, when someone shares a comment or email on certain product, then the reply come automatically because of AI mechanism.

4. Enrichment of Influencer strategies used by marketers: This AI technique is an unique technique used by different companies for influencing social media user to promote brands through online. It gsins maximum profit, if it is used properly.

5. Satisfactory customer service: Satisfaction is the major goal for any organization. For the customer satisfaction, most of the industries have used AI marketing techniques to provide them best service.

6. Improving user experience in Social Media platform: Increasing use of social media, it is difficult to handle the huge amount of unorganized data. So, top social media sites are taking the help of AI to resolve the problem.

7. Image Recognition in Marketing: Increasing number of users in social media, companies are going for online advertisement to promote their companies and brands. Increasing rate of advertisement and picture on social media to make them unique; 


\section{Role of Artificial Intelligence in Social Media and Human Behaviour}

AI technology boost every marketer to go for it due to its unique techniques to use images which helps the marketer to determine the customer behaviour.

8. Growth of competitor Analysis: Manual process is a time taking process to analyze the competitions. But the AI based analysis is time saving and accurate to find the competition chart. Also it gives some other ideas on different aspects.

9. Social sentiment Analysis: The positive or negative view of a particular post may impact on the social media site and in the review page customers are given some negative comment on that particular product. Suppose, a customer going to buy that particular item, at that moment he/she think twice to buy that product due to its negative review. To sharing the negative or positive statement on a particular post, Twitter is the most popular social site and it implement the AI to analyze and save the time of others.

Taking from the public opinions in American, based on their view on social media like YouTube, LinkedIn, Instagram and others, the Pew Research Center found 69\% people are using these social sites. Hootsuite's 2018, defined in his report on social media that media sites may use AI to personalization. Cambridge Analytica, British political consulting firm found that Russian interference in the American presidential elections over social media censorship and data privacy.

\section{Facebook}

The most popular social media network in recent period is Facebook. From a study of the School of Social Work of Washington University, whenever the user finds something has been going to be wrong on the social media posting, they can opt for Facebook to inform. Then the company gives the possible solution of that problem to the user to overcome from it. Also the user who informed about the post will contact other friends for the additional supports on a suicidal attempt. It will be a self-care advisor for needy person. The source of the below figures are web-based article.

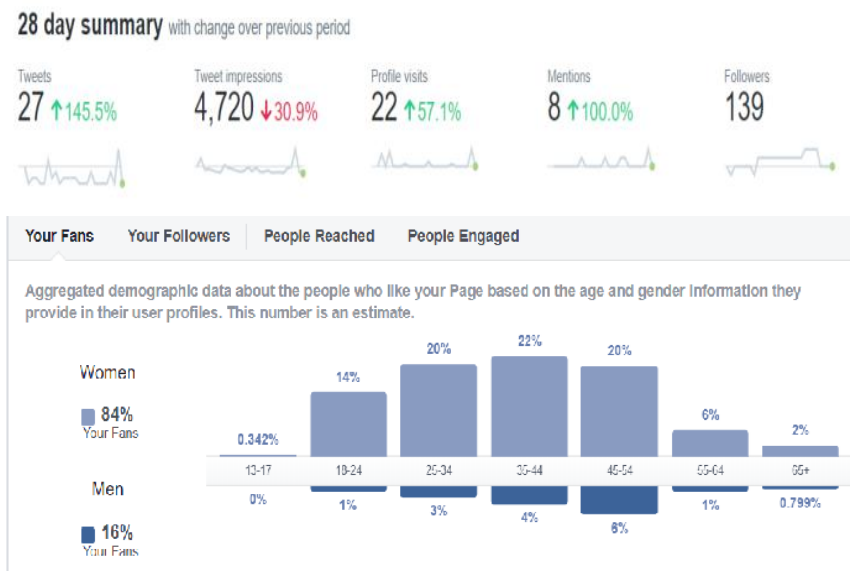

Recently, an AI research center in Montreal, Canada, opened by Facebook; where new algorithms are developed on different applications for planning and learning purpose of AI like, robotics, healthcare, games etc. According to Yann Le Cun, Director of AI, he said "Human children are quick at learning human dialogue and learning common sense about the world. We think there is something we haven't discovered yet some learning paradigm that we haven't figured out. I personally think being able to crack this nut is user. For example, on a certain product of a shopping

one of the main obstacles to making real progress in AI." [9] YouTube

YouTube is a platform where a user can find the suggestion on a product or article or even TV shows.

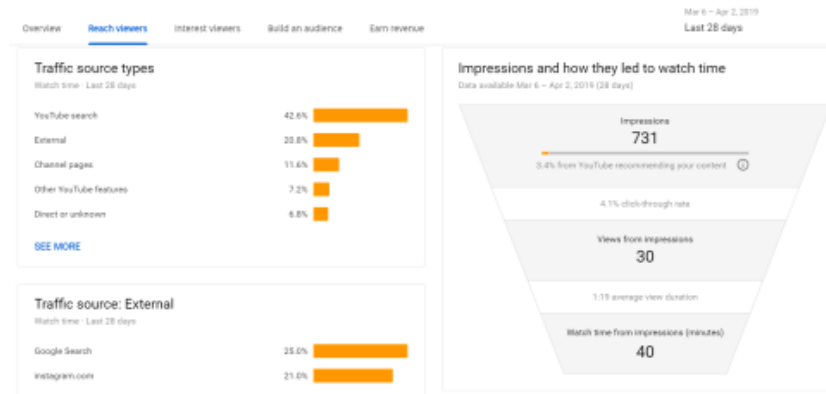

YouTube is declared as a largest video-sharing website in this world by Google. It relies on deep learning. One of the highest entertainment networking media sharing sites where users are happy and relaxed and have some time for themselves alone away from the surroundings.

\section{Twitter}

The Madbits, the computer vision start up, the acquisition was created to improve the image features in Twitter. Mr. Sephi Shapira, CEO of advertising company Massive Impact, as in his eyes to "obtain the team's talent and to apply more AI tools to advertising to build a competitive advantage against Facebook and Google." ${ }^{[9]} \mathrm{We}$ also have seen a significant number of studies on the study of a particular topic on Twitter. Twitter, particularly ensured that people were quickly informed \& updated through it. This one is the third highest social media platform for social interactions.

Table 1. Social Media user (gender wise)

\begin{tabular}{|l|l|l|l|}
\hline $\begin{array}{l}\text { Social } \\
\text { Media }\end{array}$ & Facebook & Instagram & YouTube \\
\hline Men (user) & 16 & 35 & 19.17 \\
\hline $\begin{array}{l}\text { Women } \\
\text { (user) }\end{array}$ & 84 & 65 & 23.43 \\
\hline Total in \% & 100 & 100 & 42.6 \\
\hline
\end{tabular}

\section{ARTIFICIAL INTELLIGENCE IN SOCIAL MEDIA}

The main exception is to use the location data for a relevant result. Two users from the same locality for same searching query will see the same results in most cases.AI in social media serves as source which has both personal and professional face of a person's life. AI with social media is very powerful and even misused. AI detects problems of the customers and categorized it on priority based. AI gave Stephen Hawking the ability to communicate with other by predicting the words he would use most. Interest in social media from every background such as government, private, business, voters, consumers etc. gradually changing our life style and it has been increasing rapidly from every prospective. The invention of machines is a purpose of enhancing our human and feeble capacities, by the design, they overpower our physical abilities.

Published By:

Blue Eyes Intelligence Engineering and Sciences Publication (BEIESP)

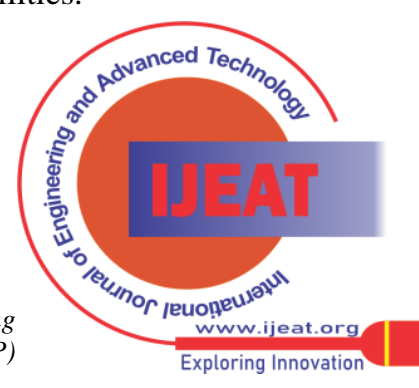


Machines give us power in terms of raw power and the computing machines provide us data generating, organizing and processing huge amounts of data at a very high speed.

\section{SOCIAL MEDIA ANALYTICS AND INTELLIGENCE RESEARCH}

Analytic research of Social media faces so many challenges. Firstly, Social media data are dynamic and huge amount of metadata due to rapidly growing use of social media by the users. Due to such things it makes lots of challenges in computing. Secondly, it contains a set of metadata which are not in systematic manner. Thirdly, it is an example of human-centered computing on social interactions. Fourthly, although it promises to tackle the noise problem with internet based information process, inconsistency issues, lack of structure, inaccuracies etc. Still, there is some lacuna to handle such problems. Using social media for future prediction in virtual organization using computational agents market approach in market research. Agents embody human-user sentiments as well as their knowledge, beliefs and assessments, all extracted from social media and participate in prediction markets to predict future events ${ }^{[5,7]}$.

\section{DISCUSSION}

This section content the evaluation of tweets, YouTube likes and viewers and facebook likes and post of different geographical locations of the followers of different gender is carried out to fulfill the objectives.

Every day at least more than 500 million tweets are tweeted which makes it virtually impossible to study all of them. Likewise, in YouTube, millions of videos are uploaded and it is not so easy to watch every videos.

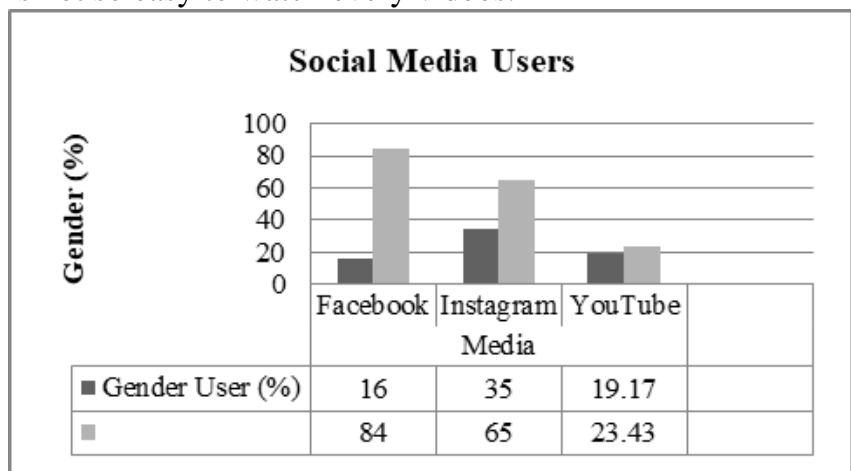

Also in facebook, everyday lots of shares and posts are posted. So, decision making process is very effective for this situation.

We are providing a comprehensive data analysis from these data. Firstly, evaluate on sample profile, validity of data and reliability of data using test for normalization, correlation and regression. Then hypothesis test is being done.

Pearson correlation indicates the relationship between two variables. This illustrates the importance of the links and the heading of the relationship to be taken as gender and social media. From this study we found that many users from Asia, North America and Europe are very active in social media and also their behaviours are different.

The figure shows the countrywide social media users in the world.

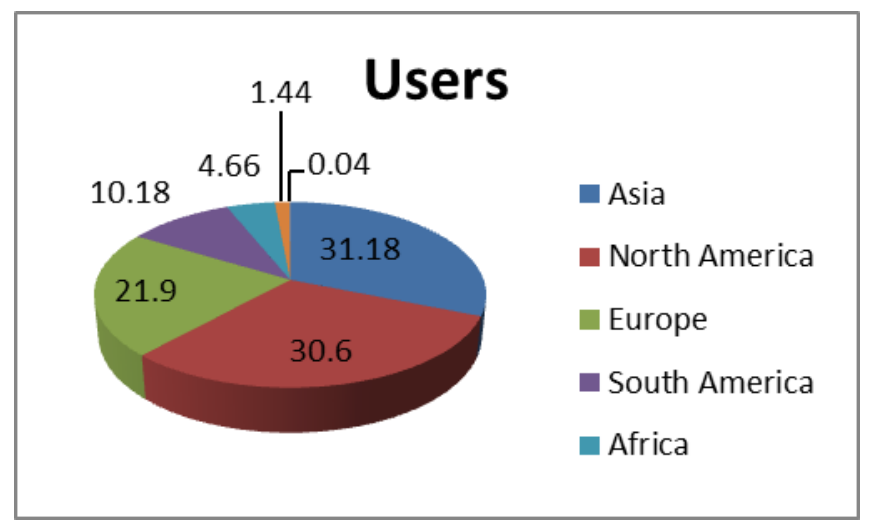

\section{CONCLUSION}

As we discuss in the above social media and the role of AI in social sites may have some limitations. To overcome from that problem we will propose a model for our future work. The paper has some survey on social media and use of $\mathrm{AI}$ in social media marketers as well as the companies. Influence of social media in our day to day life is unstoppable. To see the current situation, companies should take some necessary action on the basis of privacy and security of data. For this purpose, companies are more concern and limiting to use their information to access research freely.

\section{ACKNOWLEDGMENT}

Without the exceptional help of my supervisor, Dr. Srikanta Patnaik, this paper and research behind it would not have been feasible. He is an inspiration and kept my work on track with his passion, experience and exacting attention to detail.

\section{REFERENCES}

1. Cognitive Science and its Applications" D.Gaddam, Dr. K. Durga, T.Sandeep, TROI, vol-4, issue-1, 2017.

2. Girish kumar Jha, "ANN \& its Applications", International Journal of Computer Science \& issue, 2005.

3. M Rupai, A. Patil, "A review paper on General concept of AI \& Machine learning”, IAJSET, apecial issue-4, 2017.

4. http://techcrunch,com

5. "Social Media Analytics and Intelligent" D.Zeng, H. Chen \& R.Lsch, Shu-Hsing Li, Intelligent Systems, IEEE Computer Society, 2010

6. Smith, D. C., Cypher, A. \& Schmucker, K. 1996, 'Making programming easier for children', Interactions, vol. 3, no. 5, pp. 58-67.

7. D.D.Zeng, Hsiu-chin Chen, Robert Lusch, Shu-Hsing Li, "Social Media Analytic and Intelligent", 2011, Intelligent System, IEEE 25(6).

8. Impact of Artificial Intelligent in Social Media", An Article from https://yourstory.com/mystory/the-impact-of-artificial-intelligence-o n-social-me

9. B. Batrinca, Philip C. Treleaven, "Social Media Analytics: A survey of techniques, tools and platforms", 2015, Vol-30, Issue-1, Springer, AI \& Society.

10. "The Effect of Artificial Intelligence on Social Media", Isabella Ava, Social Media Marketing, https://thenextscoop.com

11. "Media and Artificial Intelligent", Matthew Gentzkow http://www.mediameasurement.com/mapping-influenceon-social-me dia/ Accessed 2 April 2013.

12. Bing Liu, M. Hu and M. J. Cheng: -Opinion Observer - Analyzing and comparing opinions on the Webll. Proceedings of the 14th International Conference on World Wide Web (WWW'05), Japan, pp. 342-351.

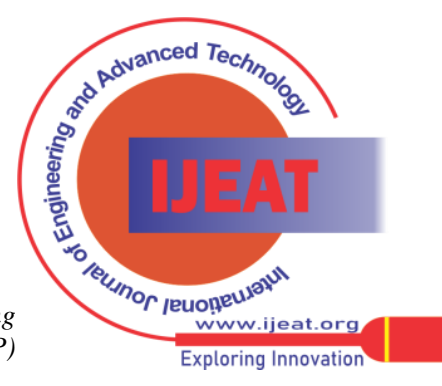


Role of Artificial Intelligence in Social Media and Human Behaviour

13. S. Baccianella, A. Esuli, and F. Sebastiani, "Multi-facet rating of product reviews," in Advances in Information Retrieval. Cham, Switzerland: Springer, 2009, pp. 461-472.

14. H. Zhang, N. Parikh2, G. Singh, N. Sundaresan, "Chelsea Won, and You Bought a T-shirt: Characterizing the Interplay Between Twitter and ECommerce", Proceedings of the IEEE/ACM International Conference on Advances in Social Networks Analysis and Mining, 2013, pp. 829836.

15. G. M. Cha, H. Haddadi, F. Benevenuto, P. Krishna, "Measuring User Influence in Twitter: The Million Follower Fallacy", Proceedings of International AAAI Conference on Weblogs and Social, 2010, pp. 10-17.

16. Lima, A. D. Silva, Sichman, S.Jaime, "SORM: A Social Opinion Relevance Model", IEEE/WIC/ACM International Joint Conferences on Web Intelligence (WI) and Intelligent Agent Technologies (IAT), 2014 2014, pp. 78 - 85.

17. Kevin Lane Keller, "Building strong brands in a modern marketing communications environment" Journal of Marketing Communications, 2006.

18. Mehrota A. \& M. Musolesi, 2017,"Sensing and modeling Human Behaviour Using Social Media and Mobile Data".

19. "Impact of Social Media on Consumer Behaviour", D. Voramontri \& L. Kileb, 2018.

\section{AUTHORS PROFILE}

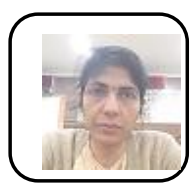

Smitarani Satpathy, doing her PhD in SOA (deemed to be) University, Bhubaneswar, India, smitasatpathy.bbsr@gmail.com. Recently, she is doing her Ph.D. under the supervision of Prof. (Dr.) Srikanta Patnaik, SOA (deemed to be) University, Bhubaneswar, Odisha. Her research interest lies in the area of machine learning and soft computing techniques. She is a sub-reviewer of IGI Global publication. She has more than 8 years of experience in teaching in different areas of computer science. She is also an Asst. editor of Graduate Research in Engineering and Technology (IRNet). She has published her research article in two IEEE conferences and also published her journal article in Scopus journal.

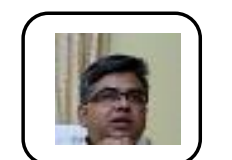

Dr. Srikanta Patnaik, he is the Director of International Publication and Relation and an eminent Professor in the department of Computer Science and Engineering, SOA(deemed to be) University, Bhubaneswar, India. and also an Editor-in-chief, Editor and Associate Editor in eight international Journals and Editor-in-Chief in Springer Book Series MOST. He is Editor of Journal of Information and Communication Convergence Engineering, published by Korean Institute of Information and Communication Convegence Engineering. He hassupervised $25 \mathrm{Ph}$. D. theses and more than 60 Master theses in the area of Computational Intelligence, Soft Computing Applications and Re-Engineering. Dr. Patnaik has published around 100 research papers in international journals and conference proceedings. 